\title{
Digital Image Processing with Two-Dimensional Haar Wavelets
}

\author{
Hakimjon Zaynidinov ${ }^{1}$, Jonibek Juraev ${ }^{2}$, Umidjon Juraev ${ }^{3}$ \\ ${ }^{1}$ Tashkent University of Information Technologies, Uzbekistan, tet2001@ @ambler.ru \\ ${ }^{2}$ Samarkand State University, Uzbekistan, jurayevju@ mail.ru \\ ${ }^{3}$ Department of Information Technology, Gulistan State University, Uzbekistan, pingo7520@gmail.com
}

\begin{abstract}
In this article an algorithm has been developed to digitally compress an image using two-dimensional Haar wavelets, reduce its size, determine the recovery coefficients, and display a higher quality image of the processed image than the original image. It is known that one of the main problems of image compression is to find and apply an effective method that allows you to present each type of pixel (dot) in a compact form. To overcome this problem, a two-dimensional Haar wavelet modification was used, and as a result, the image was compressed to make the processed image more qualitative than the existing image.
\end{abstract}

Key words: Two-dimensional Haar wavelet conversion, one-dimensional Haar wavelet conversion, image digital processing, Haar's rapid conversion algorithm, discrete signal.

\section{INTRODUCTION}

Currently, two-dimensional Haar wavelets are used in the restoration, brightening, compression of images obtained from medical devices, in image recognition, in the analysis of various images in nature (color of the eye, radiography of the kidneys, satellite images of clouds or planets, etc. possible) is used in the study of the properties of vortex fields and in other cases [2]. One of the main disadvantages of images is the lack of pixel count (low image quality) compared to the medical apparatus, and as a result, there are various difficulties in making the necessary diagnoses on X-ray images. In order to overcome these problems, the two-dimensional Haar wavelet conversion method was used $[9,10]$.

Two-dimensional Haar wavelet modification is obtained by applying one-dimensional Haar wavelet modification, i.e., two-dimensional modification is performed by processing rows and columns of the image into a onedimensional modification [1]. As a result of Haar wavelet modification of two-dimensional signals, the floating points of the signals are broken, resulting in small errors. Reducing this error depends on the approximation level of the signal[14].
A new DWT-Hungarian method of watermarking a color image was proposed in [15], and a new digital image processing algorithm was considered in [16].

\section{ONE DIMENSIONAL HAAR`S FAST CHANGE ALGORITHM}

Depending on the classes of signals, continuous and discrete wavelet modification methods are used to process them. Haar's one-dimensional wavelet rapid change is the simplest and basis for wavelet change [3,4,7]. Get $f\left(f_{1}, f_{2}, \ldots, f_{n}\right)$ one - dimensional discrete signal. As a result of discrete wavelet modification, the processed signal is divided into two pieces of equal size [5]. One is the average value view $a_{n}$ or approximation of the signal, and the other is the different value view $d_{n}$ or detail of the signal [7].

They are represented in the following form,

$$
a_{n}=\frac{f_{2 n-1}+f_{2 n}}{\sqrt{2}}, n=1,2,3, \ldots, N / 2
$$

here $a=\left\{a_{n}\right\}, n \in Z$-formula for determining the average values.

If the signal has a different value,

$$
d_{n}=\frac{f_{2 n-1}-f_{2 n}}{\sqrt{2}}, n=1,2,3, \ldots, N / 2(2)
$$

here $d_{i}=\left(d_{1}, d_{2}, \ldots, d_{N / 2}\right)$-formula for determining different values[8].

These values generate two new signals $a=\left\{a_{n}\right\}, n \in Z$ : one to restore the original signal and the other to restore the first signal $d=\left\{d_{n}\right\}, n \in Z$, indeed [6].

$$
\begin{aligned}
& f_{2 n-1}=a_{n}+d_{n} \\
& f_{2 n}=a_{n}-d_{n}
\end{aligned}
$$

If we look at the example of the rapid change wavelet sound signals (Figure 1) 


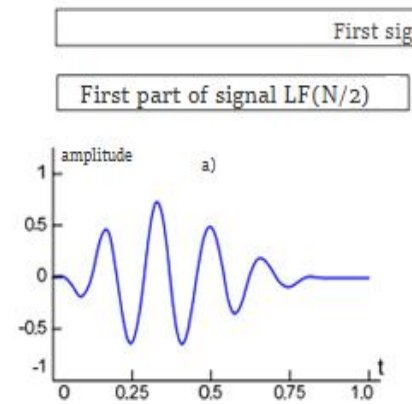

First signal(N)

Figure 1: Processing of sound signals using one-dimensional Haar wavelet, a) initial signal b) compressed signal.

To understand how the Haar Quick Change works, let's look at the following simple example [3]. Let`s suppose

$$
I=\left(\begin{array}{llll}
1 & 2 & 3 & 4 \\
4 & 5 & 6 & 7 \\
8 & 9 & 1 & 2 \\
3 & 4 & 5 & 6
\end{array}\right)
$$

When applying one-dimensional XO along the first line, the approximation coefficients are based on (1).

$$
\frac{1}{\sqrt{2}}(1+2) \text { and } \frac{1}{\sqrt{2}}(3+4)
$$

and the difference coefficients are based on (2)

$$
\frac{1}{\sqrt{2}}(1-2) \text { and } \frac{1}{\sqrt{2}}(3-4)
$$

I the massive can be applied to other rows by the same modification. By placing the approximation coefficients of each row in the first two columns and the difference coefficients corresponding to the next two columns, we obtain the following results $[8,11]$.

$$
I=\left(\begin{array}{llll}
1 & 2 & 3 & 4 \\
4 & 5 & 6 & 7 \\
8 & 9 & 1 & 2 \\
3 & 4 & 5 & 6
\end{array}\right) \stackrel{\text { on one-dimensional XO line }}{\longrightarrow}
$$

$$
\frac{1}{\sqrt{2}}\left(\begin{array}{lllll}
3 & 7 & : & -1 & -1 \\
9 & 13 & : & -1 & -1 \\
17 & 3 & : & -1 & -1 \\
7 & 11 & : & -1 & -1
\end{array}\right)
$$

In a given ratio, the approximation coefficients and the difference coefficients are separated by a dot on each line. If we apply one-dimensional XO to the column of the array generated in the next step, we have the resulting array of the first level.

$$
\frac{1}{\sqrt{2}}\left(\begin{array}{ccccc}
3 & 7 & : & -1 & -1 \\
9 & 13 & : & -1 & -1 \\
17 & 3 & : & -1 & -1 \\
7 & 11 & : & -1 & -1
\end{array}\right) \stackrel{\text { on one-dimensional XO column }}{\longrightarrow}
$$

$$
\frac{1}{2}\left(\begin{array}{ccccc}
12 & 20 & : & -2 & -2 \\
24 & 14 & : & -2 & -2 \\
\cdots & \ldots & : & \ldots & \cdots \\
-6 & -6 & : & 0 & 0 \\
10 & -8 & : & 0 & 0
\end{array}\right)
$$

So we have the following arrays,

$$
\begin{gathered}
A=\left(\begin{array}{ll}
12 & 20 \\
24 & 14
\end{array}\right), \quad H=\left(\begin{array}{ll}
-2 & -2 \\
-2 & -2
\end{array}\right) \\
V=\left(\begin{array}{cc}
-6 & -6 \\
10 & -8
\end{array}\right), \quad D=\left(\begin{array}{ll}
0 & 0 \\
0 & 0
\end{array}\right)
\end{gathered}
$$

Each massive shown in the example above has a size (number of rows/2) - (number of columns/2) and respectively they are called $\mathrm{A}, \mathrm{H}, \mathrm{V}$, and $\mathrm{D}$, [13].

A (approximation area) is the area that contains information about the global properties of the image. Deleting the spectral coefficients from this area results in the greatest distortion of the original image. $\mathrm{H}$ (horizontal area) contains information about the vertical lines hidden in the image. Removing the spectral coefficients from this area removes the horizontal details in the original image. $V$ (vertical area) contains information about the horizontal lines hidden in the image. Removing the spectral coefficients from this area eliminates the vertical details in the original image. D (diagonal area) contains information about the diagonal details hidden in the image. Removal of spectral coefficients from this field results in minimal distortions in the original image. Thus, the Quick Haar Transformation (QHT) is applied to arrays where the number of rows and columns in the image array is 2 .

Includes division into QHT'2. The number of computational operations, on the other hand, is less compared to the Haar modification (XM '). To split an image, you first need to apply a one-dimensional QHT to each line that represents the pixel value. One-dimensional QHT is applied to each column.

\section{CHANGING TWO-DIMENSIONAL HAAR WAVELET}

For example, $2 \times 2$ given massive of three-dimensional monochrome images,

$$
\left[x_{i, j}\right], i=1, \ldots, 2^{n} ; j=1, \ldots, 2^{n}(1)
$$

it can be expressed as a function of two variables $[0,1] \times[0,1]$, the part of which is defined in the unit field[8]. For a given two-variable function $f(s, t)$

$$
f(s, t)=\sum_{i=1}^{2^{n}} \sum_{j=1}^{2^{n}} x_{i, j} H_{I_{i} \times I_{j}}(s, t)(2)
$$


Let the equality be fulfilled, here

$$
\begin{gathered}
I_{i} \times I_{j}=\left[\frac{i-1}{2^{n}}, \frac{i}{2^{n}}\right] \times\left[\frac{j-1}{2^{n}}, \frac{j}{2^{n}}\right]= \\
=\left\{(s, t): s \in\left[\frac{i-1}{2^{n}}, \frac{i}{2^{n}}\right), t \in\left[\frac{j-1}{2^{n}}, \frac{j}{2^{n}}\right)\right\}
\end{gathered}
$$

and

$$
\begin{aligned}
& H_{I_{i} \times I_{j}}(s, t)=\left\{\begin{array}{l}
1,\left((s, t) \in I_{i} \times I_{j}\right) \\
0,\left((s, t) \notin I_{i} \times I_{j}\right.
\end{array}\right)= \\
& =H_{I_{i}}(s) H_{I_{j}}(t)=\frac{\phi_{n, i-1}(s)}{\sqrt{2^{n}}} \frac{\phi_{n, j-1}(t)}{\sqrt{2^{n}}}(3)
\end{aligned}
$$

The parameter s entered here is placed vertically and by substituting (3) into (2) we obtain the index $i$ of the massive $x_{i, j}[8]$.

$$
\begin{gathered}
f(s, t)=\frac{1}{2^{n}} \sum_{i=1}^{2^{n}} \sum_{j=1}^{2^{n}} x_{i, j} \phi_{n, i-1}(s) \phi_{n, j-1}(t)= \\
\frac{1}{2^{n}} \sum_{i=1}^{2^{n}}\left\{\sum_{j=1}^{2^{n}} x_{i, j} \phi_{n, j-1}(t)\right\} \phi_{n, i-1}(s)= \\
=\frac{1}{2^{n}} \sum_{i=1}^{2^{n}} z_{i}(t) \phi_{n, i-1}(s)
\end{gathered}
$$

Here

$$
z_{i}(t)=\sum_{j=1}^{2^{n}} x_{i, j} \phi_{n, j-1}(t)(5)
$$

For each I step (5) is similar to equation (3) and the first step of a one-dimensional QHT is performed [7]. We, we have a different form of equation for $z_{i}(t), \mathrm{i}=1,2, \ldots, 2^{\mathrm{n}}$ (see formula 2$)$

$$
\begin{aligned}
z_{i}(t)=\sum_{j=0}^{2^{n-1}-1} a_{n-1, j}^{i} \phi_{n-1, j}(t)+ \\
\quad+\sum_{j=0}^{2^{n-1}-1} d_{n-1, j}^{i} \psi_{n-1, j}(t)(6)
\end{aligned}
$$

Now we replace (6) with (4) and form the following

$$
f(s, t)=\frac{1}{2^{n}} \sum_{i=1}^{2^{n}} z_{i}(t) \phi_{n, i-1}(s)=
$$

$$
\begin{gathered}
\frac{1}{2^{n}} \sum_{i=1}^{2^{n}}\left(\sum_{j=0}^{2^{n}} a_{n-1, j}^{i} \phi_{n-1, j}(t)+\right. \\
\left.+\sum_{j=0}^{2^{n}} d_{n-1, j}^{i} \psi_{n-1, j}(t)\right) \phi_{n, i-1}(s)=
\end{gathered}
$$

$$
\begin{aligned}
= & \frac{1}{2^{n}}\left(\sum_{j=0}^{2^{n-1}-1}\left\{\sum_{i=1}^{2^{n}} a_{n-1, j}^{i} \phi_{n-1, j}(s)\right\} \phi_{n-1, j}(t)+\right. \\
& \left.+\sum_{j=0}^{2^{n-1}}\left\{\sum_{i=1}^{n} d_{n-1, j}^{i} \phi_{n, i-1}(s)\right\} \psi_{n-1, j}(t)\right)
\end{aligned}
$$

$$
\begin{aligned}
=\sum_{j=0}^{2^{n-1}-1} \alpha_{j}(s) \phi_{n-1, j}(t)+ \\
\\
+\sum_{j=0}^{2^{n-1}-1} \beta_{j}(s) \psi_{n-1, j}(t)(7)
\end{aligned}
$$

here

$$
\begin{gathered}
\alpha_{j}(t)=\sum_{i=0}^{2^{n}} a_{n-1, j}^{i} \phi_{n, i-1}(s) \text { and } \\
\beta_{j}(s)=\sum_{i=1}^{2^{n}} d_{n-1, j}^{i} \phi_{n, i-1}(s)
\end{gathered}
$$

for $\mathrm{j} \alpha_{j}$ and $\beta_{j}$ the expressions are constant and are similar to (3). One-dimensional QHT can be applied to it. By doing this, we get the following,

$$
\begin{gathered}
\alpha_{j}(t)=\sum_{i=0}^{2^{n}} a_{n-1, j}^{i} \phi_{n, i-1}(s)= \\
=\sum_{i=0}^{2^{n-1}-1} \tilde{a}_{n-1, i}^{j} \phi_{n-1, i}(s)+\sum_{i=0}^{2^{n-1}-1} \tilde{d}_{n-1, i}^{j} \psi_{n-1, i}(s) \\
\beta_{j}(t)=\sum_{i=0}^{2^{n}} d_{n-1, j}^{i} \phi_{n, i-1}(s)= \\
=\sum_{i=0}^{n-1}-1 \tilde{\tilde{a}}_{n-1, i}^{j} \phi_{n-1, i}(s)+ \\
+\sum_{i=0}^{n-1} \tilde{\tilde{d}}_{n-1, i}^{j} \psi_{n-1, i}(s)
\end{gathered}
$$


here

$$
\begin{gathered}
f(s, t)=\frac{1}{2^{n}}\left(\sum_{i=1}^{2^{n-1}-1} \alpha_{j}(s) \phi_{n-1, j}(t)+\right. \\
\left.+\sum_{i=1}^{2^{n-1}-1} \beta_{j}(s) \psi_{n-1, j}(t)\right)= \\
=\frac{1}{2^{n}} \sum_{j=0}^{2^{n-1}-1}\left\{2_{i=0}^{n-1} \tilde{a}_{n-1, i}^{j} \phi_{n-1, i}(s)+\right. \\
\left.+\sum_{i=0}^{n-1} \tilde{d}_{n-1, j}^{j} \psi_{n-1, i}(s)\right\} \phi_{n, j}(t)+ \\
+\sum_{j=0}^{2^{n-1}-1}\left\{2_{i=0}^{n-1} \sum_{-1}^{n-1} \tilde{\tilde{a}}_{n-1, i}^{j} \phi_{n-1, i}(s)+\right. \\
\left.\sum_{i=0} \tilde{\tilde{d}}_{n-1, j}^{j} \psi_{n-1, i}(s)\right\} \psi
\end{gathered}
$$

Considering the given equations, we get the following,

$$
\begin{gathered}
f(s, t)=\sum_{i=0}^{2^{n-1}-12^{n-1}-1} \sum_{i=1}^{n-1} \phi_{i, j} \phi_{n-1, j}(t) \phi_{n-1, i}(s)+ \\
+\sum_{i=0}^{2^{n-1}} \sum_{i=1}^{n-1} 2^{n-1} h_{i, j}^{n-1} \phi_{n-1, j}(t) \psi_{n-1, i}(s)+ \\
+\sum_{i=0}^{2^{n-1}-12^{n-1}-1} \sum_{j=0}^{n-1} \psi_{n-1, j}(t) \phi_{n-1, i}(s)+ \\
+2_{i=0}^{n-1} \sum_{i=1}^{n-1} d_{i, j}^{n-1} \psi_{n-1, j}(t) \psi_{n-1, i}(s)(8)
\end{gathered}
$$

here

$$
\begin{gathered}
a_{i, j}^{n-1}=\frac{1}{2^{n}} \tilde{a}_{n-1, i}^{j}, \quad h_{i, j}^{n-1}=\frac{1}{2^{n}} \tilde{d}_{n-1, i}^{j}, \\
v_{i, j}^{n-1}=\frac{1}{2^{n}} \tilde{\widetilde{a}}_{n-1, i}^{j}, \quad d_{i, j}^{n-1}=\frac{1}{2^{n}} \tilde{\tilde{d}}_{n-1, i}^{j}
\end{gathered}
$$

As a result, the functions are divided into the following functions [4],

$$
\begin{gathered}
\phi_{n-1, j}(t) \phi_{n-1, i}(s), \phi_{n-1, j}(t) \psi_{n-1, i}(s), \text { and } \\
\psi_{n-1, j}(t) \phi_{n-1, i}(s), \psi_{n-1, j}(t) \psi_{n-1, i}(s)
\end{gathered}
$$

Thus, the first phase of two-dimensional XM is continued by applying the first phase of one-dimensional QHT to each line of the image and then applying the first phase of one-dimensional QHT to each line of the masive .

The above (8) is two-dimensional Haar wavelet equation, which requires finding a large number of coefficients. The use of a long chain of coefficients and signal values allows to improve the quality of signal recovery.Filtering of signals is performed using two types of filters, high frequency (HF) and low frequency (LF), as shown in Figure 1. As a result, the image is divided into four parts: LFLF, LFHF, HFHF and HF. As you know, because the image is two-dimensional, filtering pixel values is done first by columns, then by rows. During the filtering process, the pixel color values are multiplied by the Haar wavelet coefficients and are the sum of the result. Thus, this conversion process continues until the last pixel of the image is etched.

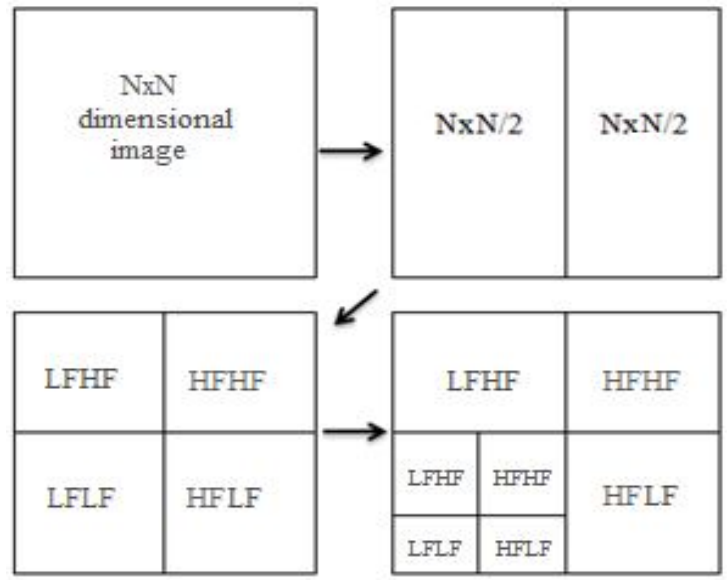

Figure 2: 1st and 2nd degree fragmentation scheme of images in two-dimensional Haar wavelet.

Suppose we were given an X-ray of the head. C ++ Builder and Matlab programs based on the model shown in (8) were used to improve the quality of the stain in that image $[11,12]$. The following results were obtained after Level 1 fragmentation and Level 2 fragmentation (Figure 3, Figure 4).

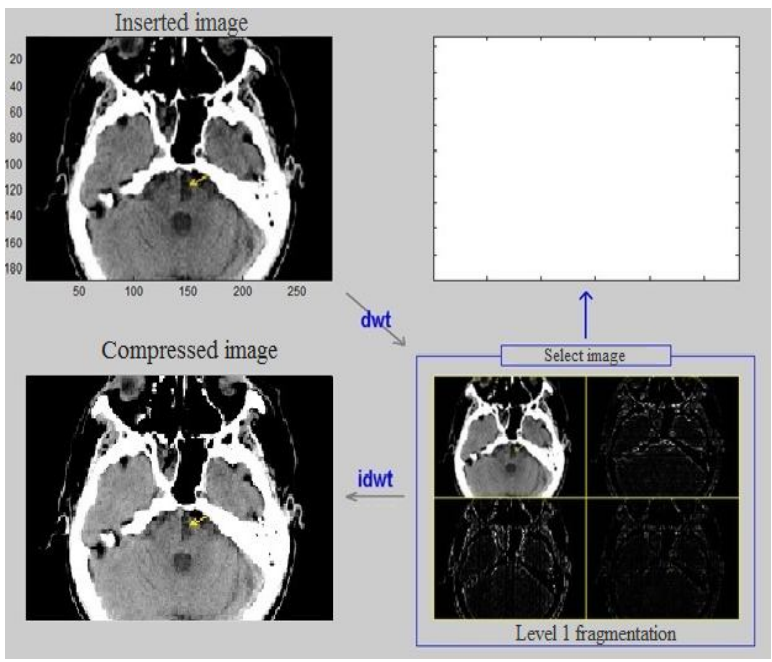

Figure 3: $1^{\text {st }}$ degree fragmentation in two-dimensional Haar wavelet. 


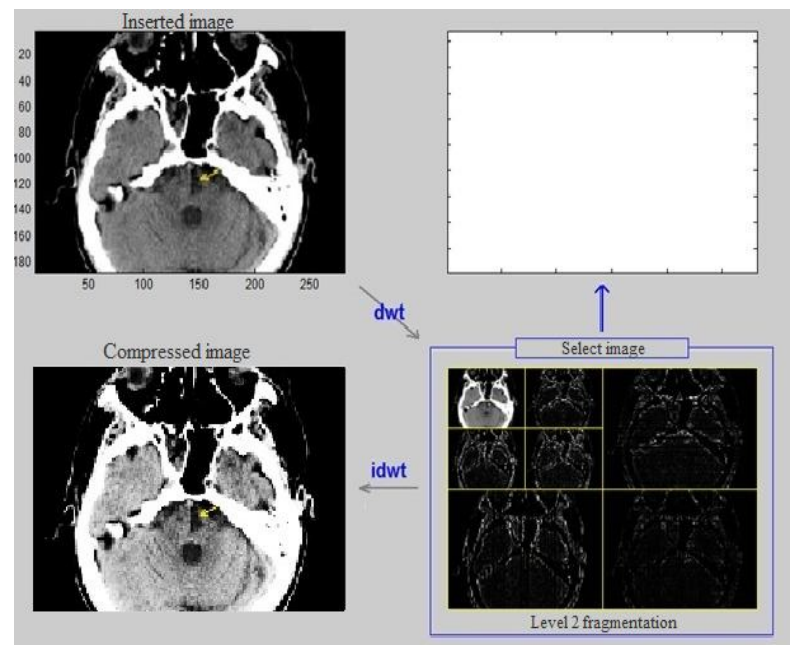

Figure 4: Level 2 fragmentation in two-dimensional Haar wavelet.

Coefficients of recovery after 1st degree fragmentation as a result of digital processing of X-ray image of the head (Table 1)

Table 1:Coefficients of recovery of the $1^{\text {st }}$ level decomposition

\begin{tabular}{|l|l|l|l|}
\hline $\begin{array}{l}\text { №-odd } \\
\text { numbers }\end{array}$ & $\begin{array}{c}\alpha_{j}(s)- \\
\text { Image } \\
\text { recovery } \\
\text { coefficients } \\
\text { (OQ) }\end{array}$ & $\begin{array}{l}\text { №- } \\
\text { couple } \\
\text { numbers }\end{array}$ & $\begin{array}{c}\beta_{j}(s) \text { - } \\
\text { Imagerecovery } \\
\text { coefficients } \\
\text { (FQ) }\end{array}$ \\
\hline $\mathbf{1 .}$ & 0 & $\mathbf{2 .}$ & 0 \\
\hline $\mathbf{3 .}$ & 0 & $\mathbf{4 .}$ & 0 \\
\hline $\mathbf{5 .}$ & 0 & $\mathbf{6 .}$ & 0 \\
\hline $\mathbf{7 .}$ & 509 & $\mathbf{8 .}$ & 507,5 \\
\hline $\mathbf{9 .}$ & 507,5 & $\mathbf{1 0 .}$ & 492,5 \\
\hline $\mathbf{1 1 .}$ & 307 & $\mathbf{1 2 .}$ & 187 \\
\hline $\mathbf{1 3 .}$ & 0 & $\mathbf{1 4 .}$ & 0 \\
\hline $\mathbf{1 5 .}$ & 437,25 & $\mathbf{1 6 .}$ & 479,75 \\
\hline $\mathbf{1 7 .}$ & 493,75 & $\mathbf{1 8 .}$ & 536,25 \\
\hline $\mathbf{1 9 .}$ & 669,5 & $\mathbf{2 0 .}$ & 824,75 \\
\hline $\mathbf{2 1 .}$ & 976 & $\mathbf{2 2 .}$ & 212,5 \\
\hline $\mathbf{2 3 .}$ & 700 & $\mathbf{2 4 .}$ & 720,75 \\
\hline $\mathbf{2 5 .}$ & 1016 & $\mathbf{2 6 .}$ & 1006,25 \\
\hline & & & \\
\hline
\end{tabular}

Coefficients of recovery after 2 nd degree fragmentation as a result of digital processing of the radiographic image of the head (Table 2)
Table 2: Coefficients of recovery of the 2nd level decomposition

\begin{tabular}{|l|l|l|l|}
\hline $\begin{array}{l}\text { No-odd } \\
\text { numbers }\end{array}$ & $\begin{array}{c}\alpha_{j}(s)- \\
\text { Image } \\
\text { recovery } \\
\text { coefficients } \\
\text { (OQ) }\end{array}$ & $\begin{array}{l}\text { oo- } \\
\text { couple } \\
\text { numbers }\end{array}$ & $\begin{array}{c}\beta_{j}(s) \text { - } \\
\text { Image } \\
\text { recovery } \\
\text { coefficients } \\
\text { (FQ) }\end{array}$ \\
\hline $\mathbf{1 .}$ & 0 & $\mathbf{2 .}$ & 0 \\
\hline $\mathbf{3 .}$ & 0 & $\mathbf{4 .}$ & 0 \\
\hline $\mathbf{5 .}$ & 0 & $\mathbf{6 .}$ & 0 \\
\hline $\mathbf{7 .}$ & 277,5 & $\mathbf{8 .}$ & 225,5 \\
\hline $\mathbf{9 .}$ & 229 & $\mathbf{1 0 .}$ & 102 \\
\hline $\mathbf{1 1 .}$ & 109 & $\mathbf{1 2 .}$ & 507 \\
\hline $\mathbf{1 3 .}$ & 0 & $\mathbf{1 4 .}$ & 0 \\
\hline $\mathbf{1 5 .}$ & 524,25 & $\mathbf{1 6 .}$ & 533 \\
\hline $\mathbf{1 7 .}$ & 489,75 & $\mathbf{1 8 .}$ & 457,75 \\
\hline $\mathbf{1 9 .}$ & 614,75 & $\mathbf{2 0 .}$ & 639,5 \\
\hline $\mathbf{2 1 .}$ & 690 & $\mathbf{2 2 .}$ & 971,5 \\
\hline $\mathbf{2 3 .}$ & 1016 & $\mathbf{2 6 .}$ & 24,25 \\
\hline $\mathbf{2 5 .}$ & 4,75 & & 1020 \\
\hline
\end{tabular}

\section{CONCLUSION}

As a result of digital processing of two-dimensional Haar wavelet conversion, an algorithm was developed based on 1st-degree and 2nd-degree compression of images, improving the quality of the compressed image and determining its recovery coefficients. As a result, the compressed image is made brighter than the existing image. The amount of these coefficients was 159048 after the 1 st degree fragmentation and 159330 after the 2nd degree fragmentation. The large amount of these coefficients give some positive results in image recovery. This developed algorithm can also be widely used in determining the number of spots in a subsequent image.

\section{REFERENCES}

1. N.M.Astafeva.Wavelet Analysis: Fundamentals of the Theory and Application Examples // Advances in Physical Sciences 1996, t.166, № 11,P. 1145- 1170 .

2. R.S. Akhmetkhanov,E.F.Dubinin, V.I.Kuksova."Application of wavelet transforms for the analysis of experimental data", Problems of mechanical engineering and automation, 2012, №. 4, P. 39-45.

3. B.Anuj, A.Rashid.Image compression using modified fast Haar wavelet transform // World 
Applied Sciences Journal. - 2009. - V. 7. -№ 5. P. 647-653.

4. R.S.Akhmetkhanov, E.F.Dubinin, V.I.Kuksova. "Application of wavelet transforms for the analysis of experimental data", Problems of mechanical engineering and automation, 2012, №. 4, P. 39-45.

5. H.N. Zaynidinov.Methods and means of signal processing in piecewise polynomial wavelets. // Tashkent, 2015. 70 p.

6. H.N.Zaynidinov.Splines in the problems of digital signal processing // Tashkent University of Information Technologies-T. "Science and technology", 2015, 208 p.

7. H.N.Zaynidinov, J.U.Juraev, M.G.Mannapova.Interpolation of functions using piecewise constant and piecewise linear Haar wavelets // Automation and Software Engineering. 2020, № 1 (31), P. 42-48.

8. H.N. Zainidinov, J.U. Juraev, U.S. Juraev.Piecewise-Polynomial HAAR Wavelets And Their Application// International Journal of Advanced Research in Science, Engineering and Technology.Vol. 7, Issue 3, March 2020, P. 13218-13224.

9. R. Gonzalez.Woods Digital image processing. M .: Techno sphere, 2005.-1072p.

10. S. Welstead.Fractals and wavelets for image compression in action.// Textbook. - M.: Publishing House Triums, 2003. - 320p.

11. N.K. Smolentsev.Fundamentals theory of wavelets. Wavelets in Matlab. - M.: DMK Press, 2005 .-- 304p.

12. Yu. E. Voskoboinikov, A. V. Gochakov, A. B. Kolker.Filtration of signals and images:Fourier and wavelet algorithms (with examples in Mathcad): monograph / Novosib. state architecture.-builds. un-t (Sibstrin). Novosibirsk: NGASU (Sibstrin), 2010 .-- 188 p.

13. G. G. Stark. The use of wavelets for DSP. - M : Techno sphere, 2007 .-- 192 p.

14. A.N. Yakovlev.Introduction to wavelet transforms. -Novosibirsk: NSTU, 2003.-- 104 p.

15. A.H.Yaribakht, M.S.Abdullah, A.Ghobadi.A novel color image watermarking method based on digital wavelet transform and hungarian algorithms.-International Journal of Advanced Trends in Computer Science and Engineering, Volume 8, Issue 2, March-April 2019, Pages 154164https://doi.org/10.30534/ijatcse/2019/0982201 9

16. B.Chinnarao,M.M.Latha.An image denoising framework based on patch grouping in complex wavelet domain. -International Journal of Advanced Trends in Computer Science and Engineering, Volume 8, Issue 5, SeptemberOctober 2019, Pages 22992306DOI: $10.30534 /$ ijatcse/2019/68852019 